\title{
National Diet and Nutrition Surveys: the British experience
}

\author{
Margaret Ashwell ${ }^{1, *}$, Susan Barlow ${ }^{2}$, Sigrid Gibson ${ }^{3}$ and Caroline Harris ${ }^{4}$ \\ ${ }^{1}$ Ashwell Associates (Europe) Ltd, Ashwell Street, Ashwell, Hertfordshire, SG7 5PZ and Oxford Brookes University, \\ Headington Campus, Gipsy Lane, Oxford OX3 OBP, UK: ${ }^{2}$ Consultant in Toxicology, 8 Harrington Road, Brighton, \\ East Sussex, BN1 6RE, UK: ${ }^{3}$ SiG-Nurture Ltd, 11 Woodway, Guildford, Surrey, GU1 2TF, UK: ${ }^{4}$ Exponent \\ International Ltd, 2D Hornbeam Park Oval, Harrogate HG2 8RB, UK
}

Submitted 19 April 2005: Accepted 31 August 2005

\begin{abstract}
Objective: The National Diet and Nutrition Surveys (NDNS) are a series of government-funded surveys of food intake, nutrient intake and nutritional status of individuals, undertaken to support nutritional policy and risk assessment. This paper summarises a review that considered the extent to which NDNS met the needs of users and suggested options for the future. The Food Standards Agency has since progressed favoured options. This paper aims to help others wishing to obtain this type of information within their own populations.

Design: A detailed questionnaire was used to probe use of data and gather opinions from users, producers and managers of the NDNS. It asked about general information needs from NDNS and changes that might be made. This was followed by a two-day workshop which included discussion of the main issues and the generation of 19 possible future options for consideration by the Agency.

Results: Options to improve effectiveness included methods to prioritise breadth and depth of coverage and possible ways of improving response and compliance. Strategies to make surveys more efficient and timely, such as adopting a rolling programme, disaggregating survey components, integrating with other studies and improving data access, were also suggested. A rolling programme, in which data are collected continuously, was the favoured option to address some of the concerns and a strategy is now in place to achieve this.

Conclusions: There is widespread support for the NDNS from its users. There is no alternative source for such high-quality data on food and nutrient consumption and nutritional status and physical measurements in the same individuals. Useful information, such as the potential value of using a rolling programme from the outset, can be gained from this British experience by others wishing to measure food and nutrient intakes and status in their own populations.
\end{abstract}

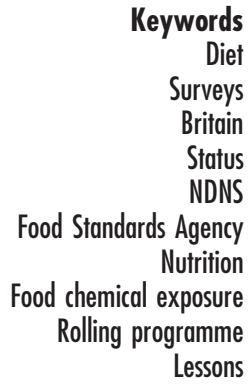

Population-wide information on food and nutrient intakes and nutrient status is essential for nutritional monitoring and surveillance and food chemical exposure assessment. It is also used to inform food and nutrition policy and healthy eating advice.

The National Diet and Nutrition Surveys (NDNS) are jointly funded by the Food Standards Agency (the Agency) and the Department of Health. The major focus of the NDNS has been to gather information to monitor the food consumption, nutrient intakes and nutritional status of the British population and for assessing exposure to food chemicals.

Many countries regard the NDNS as one of the most comprehensive in Europe. Therefore a critical review of the current programme with a constructive look to the future can help to inform those who wish to start similar surveys or those who wish to review their own methodology.

\section{Remit of the review}

In 2002, the Agency commissioned a team of scientific consultants (M.A., S.B., S.G. and C.H.) to suggest options for change to the NDNS that would best meet the information needs of the Agency in relation to food consumption, nutrient intakes, nutritional status and food chemical exposure assessment. This paper is a summary of the methodology and key findings from the project (N10015; known as REVSURVE*).

\section{NDNS and related surveys}

The NDNS programme is jointly funded and managed by the Agency and the Department of Health. The NDNS

*The full report of this project can be accessed in the library of the Agency. This comprehensive review covered the Agency's complete Dietary Surveys Programme, of which the NDNS comprises the major part. 
programme provides cross-sectional information on the dietary habits and nutritional status of nationally representative samples of the British population (see http://www.food.gov.uk/science/101717/ndnsdocuments/):

- Dietary and Nutritional Survey of British Adults (survey performed in 1986/87) ${ }^{1}$;

- National Diet and Nutrition Survey: Children aged 1.5 to 4.5 years (survey performed in 1992/93) ${ }^{2,3}$;

- National Diet and Nutrition Survey: People aged 65 years and over (survey performed in 1994/95) ${ }^{4,5}$;

- National Diet and Nutrition Survey: Young People aged 4 to 18 years (survey performed in 1997) ${ }^{6,7}$;

- National Diet and Nutrition Survey: Adults aged 19 to 64 years (survey performed in 2000/01) ${ }^{8-12}$.

Previous surveys have collected quantitative information on food consumption (weighed records over 7 days; or 4 days in refs 2 and 4), physical measurements (e.g. height, weight and blood pressure), a blood sample for analysis of nutritional status indices, a detailed interview to collect information on socio-economic, demographic and lifestyle characteristics, a physical activity record (refs 6 and 8), a urine sample (24-hour sample in refs 1 and 8), and an assessment of oral health/dental examination (refs 3, 5 and 7).

The key benefits of the NDNS are:

- detailed and robust food consumption data for individuals ( $>5000$ foods);

- information on current nutrient intakes (by combining food consumption data with the latest analyses in the Agency's nutrient databank);

- data on diet, nutritional status and related characteristics in the same individuals, to allow analysis of the links between them.

\section{Issues for consideration in the review}

The review of the dietary survey programme was prompted by four key elements.

- A lack of timeliness in the current approach. Each survey, from planning to publication, takes about 5 years. The time between collection of data on each age group does not reflect the speed of dietary changes (for example, data on adults were collected in 1986/87 and 2000/01 - a gap of some 13-15 years). This gap between data collection points limits the capacity to track changes over time and assess trends in detail.

- A lack of flexibility to respond quickly to policy needs. The long planning stage for each survey means it is not usually possible to adapt content or coverage at short notice.

- Practical issues in encouraging involvement and ensuring quality of data. Surveys are time-consuming and burdensome for respondents, and a decline in response rates has been observed for many surveys in recent years. As an illustration, the response rate to the NDNS for adults fell from $70 \%$ in $1986 / 87$ to $47 \%$ in $2000 / 01^{8}$. This has led to concerns about the representativeness of the data generated. Misreporting of food consumption is a well-recognised problem in all dietary surveys including NDNS.

- Cost-effectiveness. The cost of individual NDNS is significant and costs have risen over time as the programme has gone forward. It is necessary to ensure that the approach adopted continues to be cost-effective.

\section{Methodology of the review}

The review was based on a detailed questionnaire* followed by a two-day workshop. The questionnaire was sent electronically to 273 recipients identified as either users of the NDNS or involved in the production/ management of surveys. Completed responses were received from 103 recipients, of whom 43 were invited to the two-day workshop.

The review was assisted by an advisory panel representing NDNS users and producers from academia, industry and government, risk assessors/epidemiologists and ethicists. Their names are listed in our acknowledgements.

The Agency Board first discussed the results of the review in December 2003. Papers are available at http:// www.food.gov.uk/multimedia/pdfs/fsa031202.pdf. Minutes of the meeting are at http://www.food.gov.uk/ aboutus/ourboard/boardmeetings/board2003/

boardmeeting121103/boardminutes111203.

\section{Results and future directions}

\section{Response to REVSURVE questionnaire}

One hundred and three questionnaires were completed, a response rate of $38 \%$. Agency and other government staff comprised $16 \%$ and $13 \%$, respectively, of the final sample, while 43\% worked for academic institutions. A further 29\% were affiliated to industry or were consultants.

The distribution of non-respondents by sector was not significantly different from respondents overall $(P>0.05)$ (non-respondent sample comprised 51\% academics, 7\% and 21\% Agency and other government staff respectively, and $21 \%$ from industry). The main reason for nonresponse was that the respondent was a previous or minor user of the data or had passed the questionnaire to a colleague who was more familiar with it.

Ninety-seven per cent of questionnaire respondents agreed that NDNS is needed in Britain. Furthermore, the NDNS was considered particularly valuable in providing

*The full questionnaires can be found in the full version of the report in the Agency library. 
nationally representative data on intake and nutritional status in individuals.

\section{Options for surveys}

The review was asked to identify options for change to existing NDNS arrangements specifically to address the concerns above. Nineteen basic options were identified and 17 of these are summarised in Tables $1-3$, setting out the pros, cons and risks of adoption, and an indication of those with significant resource implications.

It was considered that abandonment of the NDNS should be rejected, as the Agency has no other source of the information needed to support food policy and protect the consumer. The option of maintaining the status quo had the support of many users but would fail to seize the opportunity to maximise the effectiveness of this important programme. Tables 1, 2 and 3 summarise the 17 other options discussed, under the headings of 'Structure of NDNS', 'Improving data quality' and 'What to include'.

Strategic options identified were: shifting the timescale of data collection to a rolling programme (Option 1), as used in the National Health and Nutrition Examination Survey (NHANES) in the USA, or using other methods to acquire food intake data (consumer panels, novel technologies) (Option 2). Reducing the volume of data collected by disaggregating the elements (Option 3) and adopting a modular survey methodology, requiring different levels of information from different people (Option 4), were also explored. These would have the advantage of reducing respondent burden and hence nonresponse bias, but would lose what some nutritionists regard as a key strength - namely the linkage of diet and nutritional status data in a high proportion of individuals. Other options explored include partial integration with the Health Survey for England (Option 5), although this might require the (NDNS-type) dietary survey to be conducted as a separate (post hoc) element.

Misreporting of food intake was acknowledged to be a widespread problem in the NDNS (as in other surveys) and threatens the reliability of the data and estimates derived from it. It was considered essential that research be conducted into both prevention (i.e. ways of minimising) (Option 9) and cure (i.e. post hoc treatments such as modelling and the use of biomarkers) (Option 10). Whilst no method is likely to eliminate misreporting entirely, this could at least allow some quantification of the errors and hence uncertainty surrounding estimates of nutrition and exposure.

The breadth (i.e. population coverage) and depth (i.e. detail) of the surveys were generally considered adequate, although suggestions were made for improvements and possible methods for prioritising needs (Options 12-16). There was general agreement that respondent burden was high, leading to poor response rates, and this was probed by discussing some of the underlying issues such as incentives (Option 6), ethical approval process (Option 7), survey methodology (Option 8) and improved promotion of the importance of taking part in NDNS (Option 11). In fact, dissemination is likely to be fundamental to the future of the NDNS, essential to encourage greater public awareness and respondent participation and to maximise the fruitful exploitation of the data.

\section{Future directions for the NDNS}

Of the options, it was clear that the rolling programme (Option 1) was the only one that addressed the issues of timeliness and flexibility that prompted the review. A rolling programme would address the issue of lack of timeliness in the current arrangements as, once established, it would generate data on a continuous basis, strengthening the ability to track changes over time and avoiding long gaps between data collection points. As data would be collected every year there would be the added flexibility to add targeted studies (such as adjunct surveys and 'bolt-ons') at relatively short notice, making the programme more responsive to immediate policy needs. None of the other options addressed these issues. The continuous nature of the rolling programme approach might also raise the profile of the survey, which might improve response rates. Survey management would be more cost-effective, eliminating the need for repeated planning, set-up costs and feasibility studies, and allowing evaluation at any stage.

The Agency's Board agreed in principle in December 2003 to move to a rolling programme format for future dietary surveys, subject to seeing more detailed proposals. Proposals were drawn up during 2004 following informal discussions with a range of stakeholders. The Board approved the proposals for the core rolling programme, subject to availability of funding, at its meeting in February 2005. The proposals for structure, content and funding for the new programme have now been published on the Agency website at http://www.food.gov.uk/multimedia/ pdfs/fsa050203.pdf. Key stakeholder organisations have indicated support for a move towards a good-quality rolling programme approach that builds on the track record of the NDNS and gives scope for monitoring trends.

The proposed rolling programme will provide data for risk assessment and be the primary method for monitoring progress against nutrition targets in the Agency's Strategic Plan 2005-2010. It could also be used to monitor progress on diet and nutrition objectives set out in the White Paper 'Choosing Health'. The programme will be designed to be representative of the total UK population, now including Northern Ireland. People living in institutions such as residential homes for the elderly, prisons and hospitals are not covered by the proposed programme. The rolling programme offers opportunities for enhancement by sample boosts in specific regions or groups, and by bolt-on surveys and additional components to provide indepth focus on special issues. 


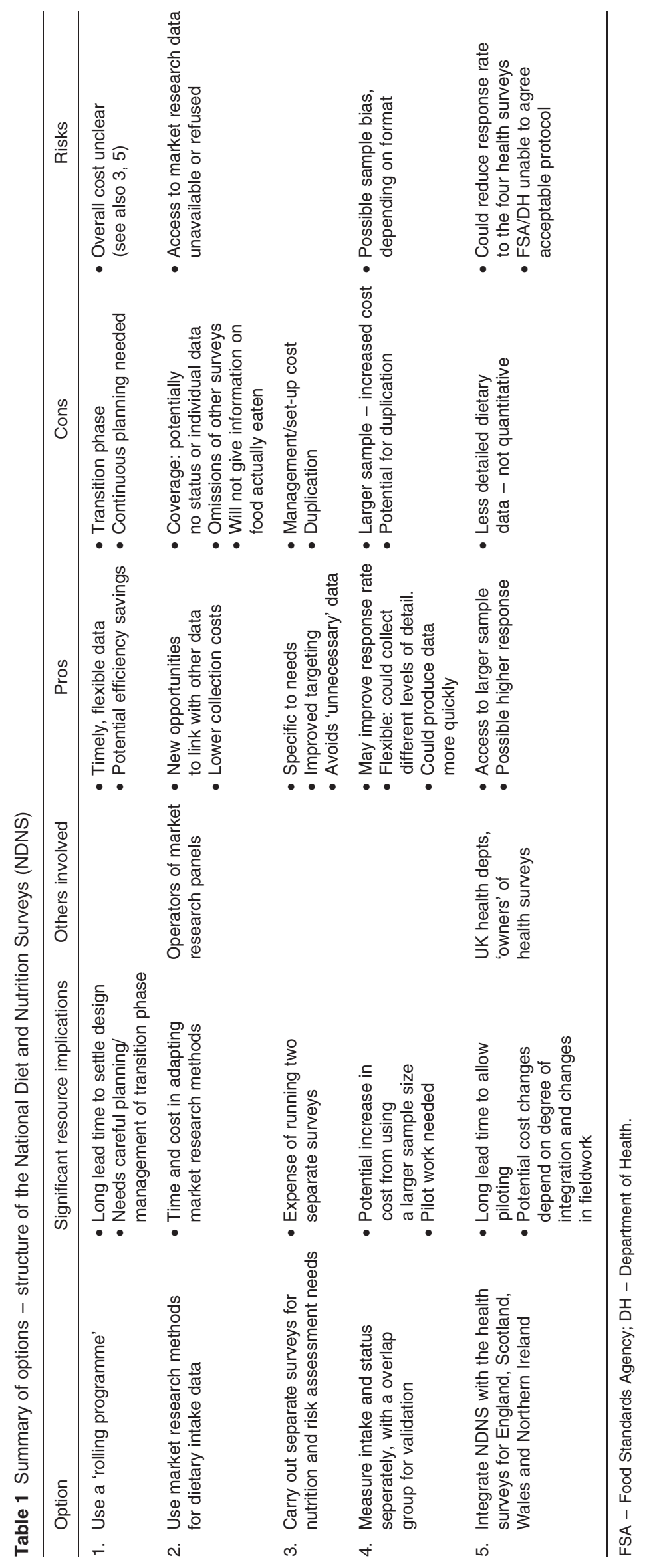




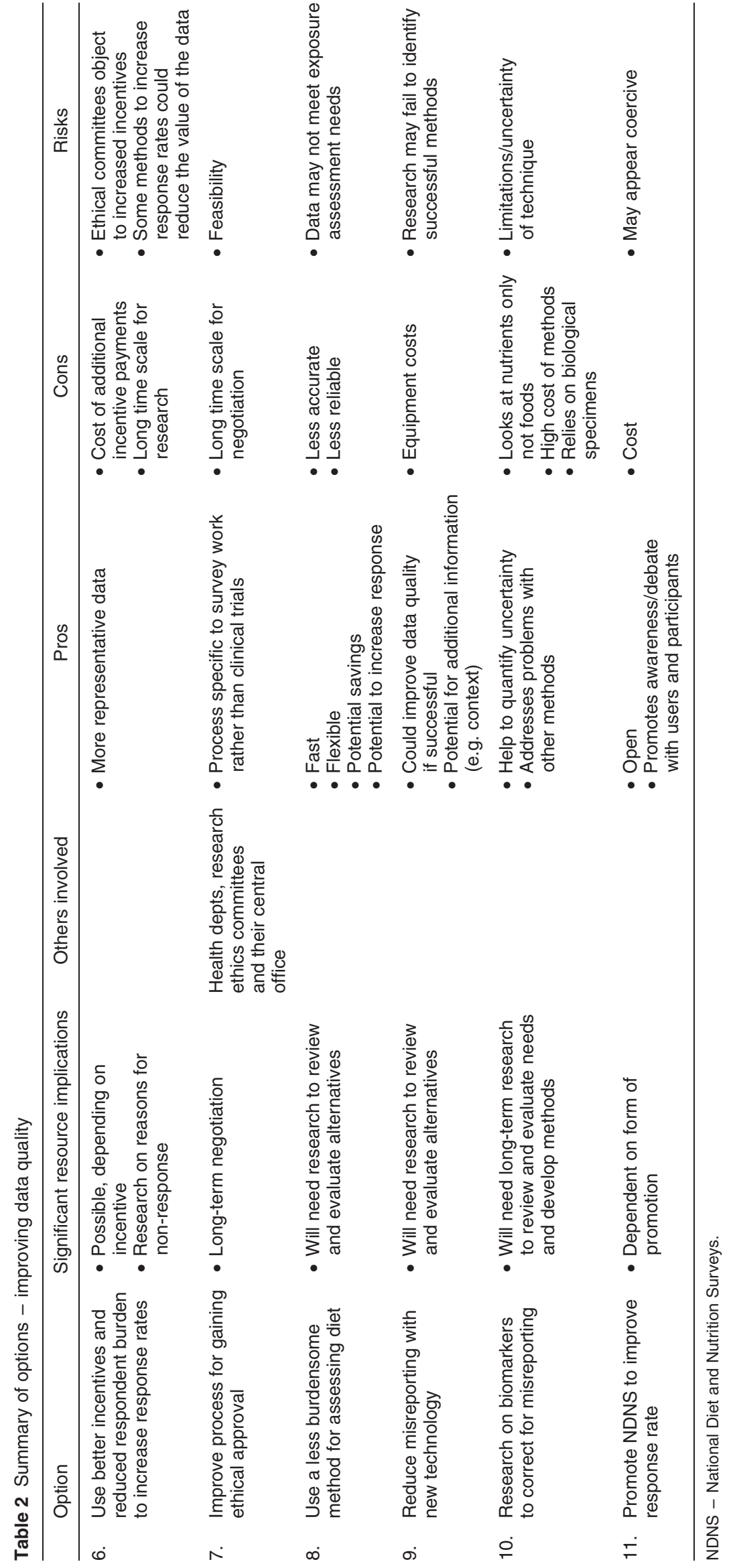




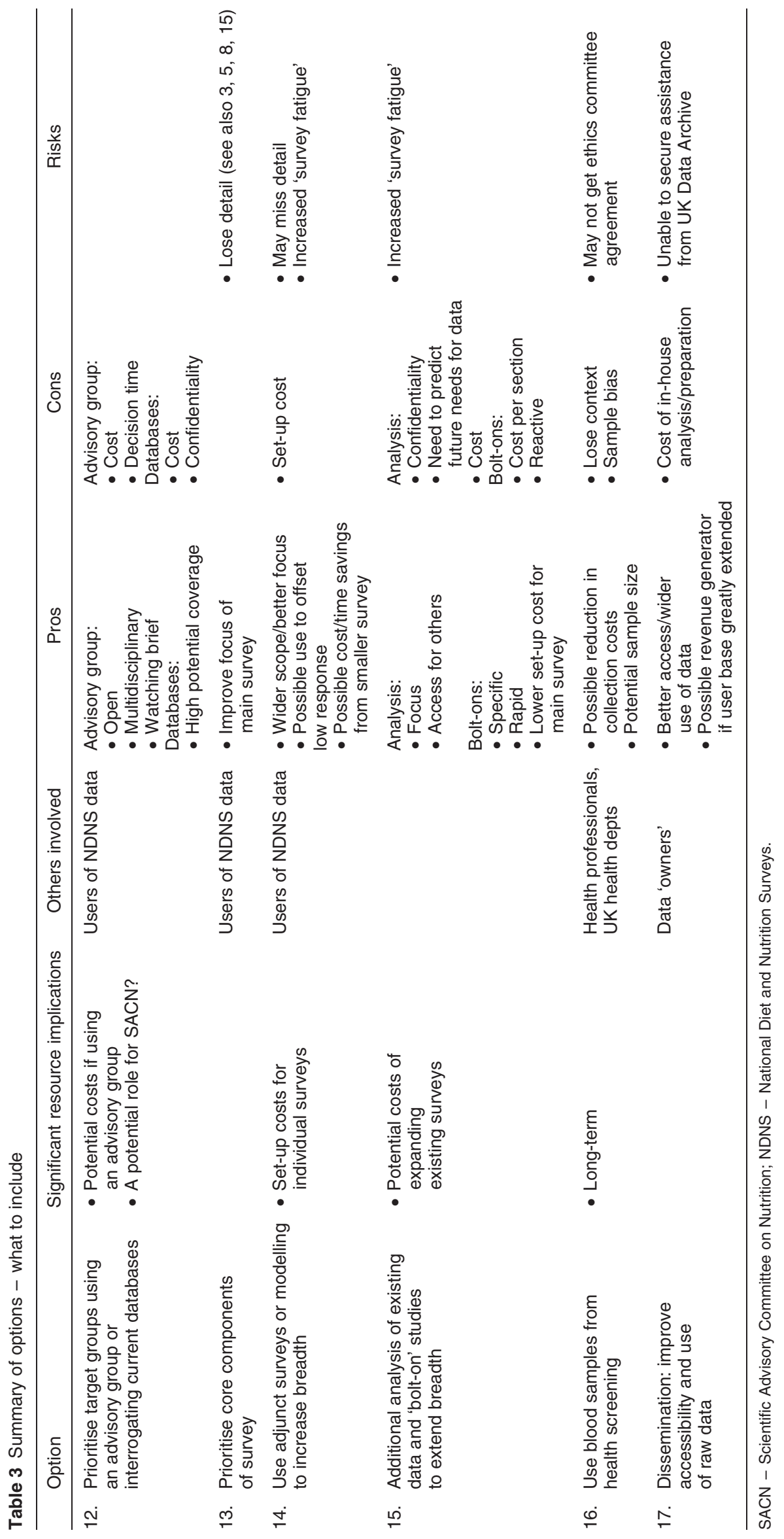


Proposals are based on a sample size of 1000 people per year for the core programme, covering both adults and children (aged 1.5 years upwards). This would allow a group of 1500 adults or children to build up over two years for analysis. This sample size and structure would generate data on food and nutrient intakes sufficient for analysis every $1-3$ years and on nutritional status and salt intakes every $4-5$ years. Dietary survey assessment would be included for all participants on an annual basis, blood samples and 24-hour urine collections less frequently or in a sub-sample. The dietary assessment method proposed is based on the multiple-pass 24-hour dietary recall repeated on four non-consecutive days. This is considered to produce data of comparable quality to the weighed record but be much less burdensome for participants. It will be tested alongside the existing weighed intake method to ensure that the method can be compared with data generated in earlier surveys. The Agency is also developing proposals to address the problem of underreporting and is considering methods to improve response rates such as increasing the token of appreciation. The Agency is pursuing discussions with health departments, devolved administrations and external organisations to identify co-funding for the core programme and for proposed enhancements to boost sample sizes in ethnic minority groups and in the devolved countries.

Provided funding is secured, pilot study fieldwork will commence in 2006 with the first phase of the new rolling programme to commence in 2007.

\section{Conclusions}

There is a very high level of support for the NDNS from a wide range of users, who would have no alternative source for such high-quality nationally representative data on food and nutrient intakes, or data on nutritional and physical status in the same individuals.

Nineteen options to improve effectiveness were suggested to the Agency. These included methods to prioritise breadth and depth of coverage and possible means of improving response and compliance. Strategies to make surveys more efficient and timely such as adopting a rolling programme, disaggregating survey components, integrating with other studies and improving data access are also suggested. A move to a 'rolling programme' for the NDNS was the favoured option to address some of the concerns and a strategy is now in place to achieve this.

\section{Value of NDNS review for other countries}

Although this review was conducted on the British NDNS programme, this is widely acknowledged to be the most advanced within Europe. This view was confirmed by the international respondents to the questionnaire $(n=19$, representing 13 countries). The central skill base in Britain was one of the key reasons identified as leading to the high quality of the NDNS compared with surveys conducted outside Britain. Many Britain surveys are conducted at household level which does not allow for the precision required for analysis of nutritional status and risk assessment.

Many of the international respondents considered that their national surveys gave insufficient information on food consumption, while most felt they did not provide adequate information on nutritional status from blood and urine analytes.

Lack of timeliness and difficulty in monitoring trends are problems in several countries. Only the US NHANES and the Danish food consumption study use a rolling programme approach to monitor trends. Comparison between cross-sectional surveys conducted several years apart is made in other countries, but comparability of methods is key. Efforts in the future could be directed to collecting data that could be maintained as part of a harmonised European database (as proposed by the EFCOSUM initiative).

Other countries may be able to benefit from this review: not only from how the NDNS have been conducted in the past, but also on some possible solutions to the problems currently facing the British programme, e.g. methods to prioritise breadth and depth of coverage and possible ways of improving response and compliance. New British strategies to make surveys more efficient and timely such as adopting a rolling programme, disaggregating survey components, integrating with other studies and improving data access could be included in new programmes right from the outset.

\section{Acknowledgements}

We are grateful for assistance from all those who completed our questionnaire and to our advisory panel: Dr Chris Bates (MRC-HNR, Cambridge) Dr Janet Cade (University of Leeds), Dr Ruth Chadwick (Lancaster University), Dr Judy Cunningham (ANZFA, Australia), Dr Wendy Doyle (British Dietetic Association), Ms Jan Gregory (Office for National Statistics), Dr Elaine Gunter (Centers for Disease Control and Prevention, USA), Mr Paul Hamey (Pesticides Safety Directorate), Ms Jenny Kemp (Taylor Nelson Sofres), Dr Paul Nestel (Australia), Mrs Barbara Saunders (consumer consultant), Dr David Shuker (Open University), Dr Philippe Verger (INRA, France), Professor Martin Wiseman (independent consultant).

We are also grateful to all the Agency officials with expertise in nutrition, exposure assessment and statistics who were involved in this review.

This project was funded by the Agency, contract number N10015. A full report of the project is available from the Agency library. 


\section{References}

1 Gregory J, Foster K, Tyler H, Wiseman M. The Dietary and Nutritional Survey of British Adults. London: HMSO, 1990.

2 Gregory J, Collins D, Davies P, Hughes J, Clarke P. National Diet and Nutrition Survey: Children aged 1.5 to 4.5 years Vol. 1. Report of the Diet and Nutrition Survey. London: HMSO, 1995.

3 Hinds K, Gregory J. National Diet and Nutrition Survey: Children aged 1.5 to 4.5 years. Vol. 2. Report of the Dental Survey. London: HMSO, 1995.

4 Finch S, Doyle W, Lowe C, Bates C, Prentice A, Smithers G, et al. National Diet and Nutrition Survey: People aged 65 years and over. Vol. 1. Report of the Diet and Nutrition Survey. London: The Stationery Office, 1998.

5 Steele J, Sheiham A, Marcenes W, Walls AWG. National Diet and Nutrition Survey: People aged 65 years and over. Vol. 2. Report of the Oral Health Survey. London: The Stationery Office, 1998.

6 Gregory J, Lowe S. National Diet and Nutrition Survey (NDNS): Young people aged 4-18 years. London: The Stationery Office, 2000

7 Walker A, Bradnock GJ, Nunn J, White D. National Diet and Nutrition Survey: Young people aged 4 to 18 years. Vol. 2.
Report of the Oral Health Survey. London: The Stationery Office, 2000

8 Henderson L, Gregory J, Swan G. National Diet and Nutrition Survey (NDNS): Adults aged 19-64 years. Vol. 1. Types and Quantities of Foods Consumed. London: The Stationery Office, 2002.

9 Henderson L, Gregory J, Irving K, Swan G. The National Diet and Nutrition Survey: Adults aged 19 to 64 years. Vol. 2. Energy, Protein, Carbohydrate, Fat and Alcohol Intake. London: The Stationery Office, 2003.

10 Henderson L, Irving K, Gregory J, Bates CJ, Prentice A, Perks J, et al. The National Diet and Nutrition Survey: Adults aged 19 to 64 years. Vol. 3. Vitamin and Mineral Intake and Urinary Analytes. London: The Stationery Office, 2003.

11 Ruston D, Hoare J, Henderson L, Gregory J, Bates CJ, Prentice A, et al. The National Diet and Nutrition Survey: Adults aged 19 to 64 years. Vol. 4. Nutritional Status (Anthropometry and Blood Analytes), Blood Pressure and Physical Activity. London: The Stationery Office, 2004.

12 Hoare J, Henderson L, Bates CJ, Prentice A, Birch M, Swan G, et al. The National Diet and Nutrition Survey: Adults aged 19 to 64 years. Vol. 5. Summary Report. London: The Stationery Office, 2004. 